EDITOR'S

CHOICE

\title{
Electrical and mechanical dyssynchrony for prediction of cardiac events in patients with systolic heart failure
}

\author{
Goo-Yeong Cho, ${ }^{1}$ Hyung-Kwan Kim, ${ }^{2}$ Yong-Jin Kim, ${ }^{2}$ Dong-Ju Choi, ${ }^{1}$ Dae-Won Sohn, ${ }^{2}$ \\ Byung-Hee Oh, ${ }^{2}$ Young-Bae Park ${ }^{2}$
}

${ }^{1}$ Division of Cardiology, Seoul National University Bundang Hospital, Seoul National University, Seoul, South Korea DDivision of Cardiology, Seoul National University Hospital, Seoul National University, Seoul, South Korea

\section{Correspondence to} Professor Goo-Yeong Cho, Division of Cardiology, College of Medicine, Seoul National University and Cardiovascular Centre, Seoul National University Bundang Hospital, 300 Gumi-dong, Bundang-gu, Seongnam-si, Gyeonggi-do, 463-707, South Korea; cardioch@medimail.co.kr

Accepted 20 October 2009 Published Online First 11 November 2009

\begin{abstract}
Background Recent clinical trials have challenged the clinical applicability of mechanical dyssynchrony in predicting cardiac resynchronisation therapy response. Objective To evaluate whether mechanical dyssynchrony has an additional benefit over ORS duration in predicting cardiac events in patients with systolic heart failure.

Methods A total 167 patients admitted to hospital with heart failure (age $65 \pm 12$, ejection fraction $<35 \%$ ) were followed up prospectively. Using tissue Doppler imaging (TDI), the time to peak systolic velocity during the ejection phase was measured in the basal septal and lateral segments. A temporal difference between the septal to lateral wall (Ts-I) of $\geq 65 \mathrm{~ms}$ was defined as a mechanical dyssynchrony.
\end{abstract}

Results After 33 months of follow-up, 70 patients (41.9\%) had cardiac events, including 42 (25.1\%) with cardiac death. The event-free survival time decreased as Ts-I or QRS duration increased. Patients with QRS $\geq 120 \mathrm{~ms}$ had increased risks of cardiac events by multivariate Cox proportional hazard analysis $(\mathrm{HR}=1.88$, $95 \% \mathrm{Cl} 1.07$ to $3.29, p=0.028)$. The presence of mechanical dyssynchrony also predicted an increased risk of cardiac events $(\mathrm{HR}=2.37,95 \% \mathrm{Cl} 1.39$ to 4.04 , $p=0.002)$. Those with both electrical and mechanical dyssynchrony had a HR of $3.98(95 \% \mathrm{Cl} 2.02$ to 7.86 , $\mathrm{p}<0.001)$ when compared with those with normal QRS duration and absence of mechanical dyssynchrony. The addition of mechanical dyssynchrony significantly improved the prognostic power of a model containing echocardiographic parameters and QRS duration.

Conclusions TDI-derived mechanical dyssynchrony is an important prognosticator and independently associated with QRS duration in predicting adverse events in patients with systolic heart failure.

\section{INTRODUCTION}

An intraventricular conduction delay manifested by wide ORS complex in patients with heart failure is associated with advanced myocardial disease, decreased left ventricular (LV) function, and a poor prognosis. ${ }^{1}$ Because of this, cardiac resynchronisation therapy (CRT) has been recommended for patients with symptomatic heart failure of New York Heart Association (NYHA) functional class $\geq$ III and who show evidence of electrical dyssynchrony. However, there is a general consensus that a wide ORS complex has only a limited relationship with intraventricular mechanical dyssynchrony and may underestimate intraventricular dyssynchrony. ${ }^{2-4}$ Echocardiography has had a key role in evaluating mechanical dyssynchrony, with tissue Doppler imaging (TDI) being the most widely used screening tool. ${ }^{5-9}$ However, results of recent clinical trials have challenged the applicability of mechanical dyssynchrony using echocardiography in 'realworld' clinical practice. ${ }^{10-12}$ We sought to determine whether mechanical dyssynchrony had an additional benefit over ORS duration in predicting cardiac events in patients with systolic heart failure.

\section{MATERIALS AND METHODS \\ Study population}

Our study is a single-centre, prospective observational study. Patients who were hospitalised for NYHA functional class $\geq$ III heart failure, caused by either ischaemic or non-ischaemic events, and LV ejection fraction (LVEF) $\leq 35 \%$ were consecutively enrolled. Of these, we excluded patients aged $\geq 80$ other than sinus rhythm, cardiac or cerebral ischaemic events within the past 3 months or coexisting serious illnesses. Patients who underwent coronary revascularisation during the study period were also excluded. A total of 167 patients were included in this study. Unless contraindicated, patients were treated with $\beta$ blockers or ACE inhibitors (or angiotensin receptor blockers) and diuretics at the time of discharge, and the dose of $\beta$ blocker and ACE inhibitors was increased to maximal tolerable dose. Clinical events included hospitalisation for worsening of heart failure and cardiac death. Cardiac death was verified from hospital records or death certificate from the primary doctors. The study protocol was approved by the institutional review board of our university.

\section{Standard and tissue Doppler echocardiography}

Standard echocardiography was performed using a commercially available system (Vivid 5 or 7; General Electric, Norway). LV dimensions were measured by $\mathrm{M}$-mode echocardiography according to the guidelines of the American Society of Echocardiography. LV end-systolic and end-diastolic volumes and LVEF were measured using the modified biplane Simpson rule. Mitral regurgitation was characterised as follows: mild (regurgitation orifice area $\left.<0.2 \mathrm{~cm}^{2}\right)$, moderate $\left(0.2-0.39 \mathrm{~cm}^{2}\right)$ and severe $\left(\geq 0.4 \mathrm{~cm}^{2}\right)$.

For TDI, colour Doppler frame rates varied between 99 and 130 frames/s depending on the sector width. Three cardiac cycles triggered to the ORS complex were stored in digital format on a magneto-optical disk for offline analysis (EchoPac BT07; General Electric). 


\section{Ventricular dyssynchrony}

To assess regional myocardial velocity, the sample volume was placed in the basal portions of the septum and lateral wall; the time to peak systolic velocity from the $\mathrm{R}$ wave on ORS complex during the ejection period was measured and expressed in milliseconds. LV dyssynchrony was derived from the time difference between the septum and lateral wall $(\mathrm{Ts}-\mathrm{l}) .^{13}$ If the peak velocity could not be defined because of the noise signal or flat velocity contour, the sample volume was gradually moved to mid-segment until a clear signal intensity could be obtained. We divided patients into four groups based on their ORS duration and the presence or absence of mechanical dyssynchrony; group I: QRS duration $<120 \mathrm{~ms}$ and $\mathrm{Ts}-1<65 \mathrm{~ms}, \mathrm{n}=71$, group II: ORS duration $\geq 120 \mathrm{~ms}$ and $\mathrm{Ts}-1<65 \mathrm{~ms}, \mathrm{n}=25$, group III: ORS duration $<120 \mathrm{~ms}$ and $\mathrm{Ts}-1 \geq 65 \mathrm{~ms}, \mathrm{n}=44$, group IV: ORS duration $\geq 120 \mathrm{~ms}$ and $\mathrm{Ts}-1 \geq 65 \mathrm{~ms}, \mathrm{n}=27$.

\section{Statistics}

Summary data are expressed as mean value \pm SD or percentage of patients. Comparison of baseline characteristics between the groups was performed with independent $t$ test and $\chi^{2}$ test. The area under the receiver operating characteristic curve (AUC) was used to compare the predictive validity. A z score was calculated to determine the difference between AUCs. Survival curves were estimated by the Kaplan-Meier method with a log rank test. Predictive variables for cardiac events or cardiac death were examined using the univariate or multivariate Cox proportional hazard regression models. A p value $<0.05$ was considered statistically significant.

\section{RESULTS}

\section{Patients characteristics}

Of 167 patients, 52 patients had a ORS $\geq 120 \mathrm{~ms}$ (31.1\%) and 71 patients had a $\mathrm{Ts}-1 \geq 65 \mathrm{~ms}$ (42.5\%). After a mean follow-up of $33.4 \pm 19.9$ months, 70 patients $(41.9 \%)$ had cardiac events, including cardiac death in 42 patients $(25.1 \%)$. Baseline clinical and echocardiographic characteristics are summarised in table 1.

\section{Reproducibility}

The reproducibility was determined on a single set of recordings. Variability in the measurement of mechanical dyssynchrony (Ts-1) was evaluated in 20 randomly selected patients. For intraobserver variability, the same observer measured Ts-1 for each of the selected patients again 15 days later. For the interobserver variability, a second independent observer repeated the analysis. The coefficients of variations of intra- and interobserver variability were $9.1 \%$ and $10.1 \%$, respectively.

\section{Predictors of cardiac events}

In univariate analysis, older age, ischaemic aetiology, longer QRS duration, no $\beta$-blocker use and longer Ts -1 were associated with cardiac events. In multivariate Cox proportional hazard analysis, both $\mathrm{ORS}$ duration $(\mathrm{HR}=1.88, \mathrm{p}=0.028)$ and $\mathrm{Ts}-1(\mathrm{HR}=2.37$, $\mathrm{p}=0.002)$ were independent predictors of cardiac events (table 2). Those with both electrical and mechanical dyssynchrony had a HR of 3.98 (95\% CI 2.02 to 7.86, p <0.001) when compared with those with normal QRS duration and an absence of mechanical dyssynchrony. The additional benefit of mechanical dyssynchrony in the prediction of cardiac events is shown in figure 1 . The addition of mechanical dyssynchrony significantly improved the prognostic power of a model containing echocardiographic parameters (LVEF and E/e') and ORS duration. We calculated the AUCs of ORS duration and $\mathrm{Ts}-1$ for predicting cardiac events. The AUC of Ts-1 was 0.62 ,
Table 1 Baseline clinical and echocardiographic parameters

\begin{tabular}{|c|c|c|c|c|}
\hline & \multirow[b]{2}{*}{ All patients } & \multicolumn{2}{|l|}{ Clinical event } & \multirow[b]{2}{*}{ p Value } \\
\hline & & No $(n=97)$ & Yes $(n=70)$ & \\
\hline Age & $64.6 \pm 11.8$ & $63.3 \pm 11.5$ & $66.5 \pm 11.9$ & 0.092 \\
\hline Sex (male, \%) & 65.3 & 67.0 & 62.9 & 0.623 \\
\hline \multicolumn{5}{|l|}{ Surface ECG } \\
\hline QRS duration & $112.5 \pm 27.1$ & $108.2 \pm 23.8$ & $118.5 \pm 30.2$ & 0.019 \\
\hline LBBB (\%) & 16.2 & 12.4 & 21.4 & 0.138 \\
\hline Ischaemic origin & $98(58.7 \%)$ & $48(49.5 \%)$ & $50(71.4 \%)$ & 0.007 \\
\hline Diabetes mellitus (\%) & 39.2 & 33.3 & 47.9 & 0.079 \\
\hline Creatinine (mg/dl) & $1.78 \pm 1.78$ & $1.44 \pm 1.42$ & $2.19 \pm 2.07$ & 0.010 \\
\hline \multicolumn{5}{|l|}{ Drugs } \\
\hline$\beta$ Blocker (\%) & 79 & 85.3 & 72.9 & 0.075 \\
\hline $\begin{array}{l}\text { Dose of carvedilol, } \\
\text { (mg) }\end{array}$ & $30.7 \pm 13.3$ & $33.3 \pm 13.5$ & $27.3 \pm 12.4$ & 0.022 \\
\hline \multirow[t]{2}{*}{ ACEI or ARB (\%) } & 92.8 & 95.8 & 90.0 & 0.205 \\
\hline & 86.2 & 84.4 & 90.0 & 0.211 \\
\hline \multicolumn{5}{|l|}{ LV dimension (mm) } \\
\hline End-diastole & $61.0 \pm 8.2$ & $60.7 \pm 6.9$ & $61.3 \pm 9.8$ & 0.653 \\
\hline End-systole & $49.5 \pm 9.8$ & $49.4 \pm 8.7$ & $49.5 \pm 11.1$ & 0.966 \\
\hline \multicolumn{5}{|l|}{ LV volume (ml) } \\
\hline End-diastole & $145.5 \pm 55.8$ & $139.4 \pm 52.2$ & $154.0 \pm 59.7$ & 0.103 \\
\hline End-systole & $101.8 \pm 47.4$ & $102.8 \pm 42.1$ & $115.5 \pm 53.2$ & 0.101 \\
\hline LV ejection fraction (\%) & $26.8 \pm 6.5$ & $27.2 \pm 6.5$ & $26.3 \pm 6.3$ & 0.373 \\
\hline Mitral regurgitation (\%) & & & & 0.032 \\
\hline Mild & $46 \%$ & $51.1 \%$ & $39.1 \%$ & \\
\hline Moderate & $36.8 \%$ & $38.3 \%$ & $34.8 \%$ & \\
\hline Severe & $17.2 \%$ & $10.6 \%$ & $26.1 \%$ & \\
\hline Ts-I (ms) & $64.2 \pm 48.2$ & $55.0 \pm 42.5$ & $76.3 \pm 52.8$ & 0.005 \\
\hline
\end{tabular}

Mitral regurgitation: mild (regurgitation orifice area $\left.\leq 0.2 \mathrm{~cm}^{2}\right)$, moderate $\left(0.2-0.39 \mathrm{~cm}^{2}\right.$ ), and severe $\left(\geq 0.4 \mathrm{~cm}^{2}\right)$.

ACEI, ACE inhibitor; ARB, angiotensin receptor blocker; LBBB, left bundle branch block; LV left ventricular; Ts-I, time difference between the septal and lateral wall.

which is not statistically different from that of ORS duration (0.60) (z score $=0.31, \mathrm{p}=0.76$ ).

\section{Kaplan-Meier analysis}

Cardiac events during follow-up were 23.9\% (Kaplan-Meier estimate; 17 events in 71 patients) in group I, 40.0\% (10/25) in group II, $59.1 \%(26 / 44)$ in group III, and $63.0 \%(17 / 27)$ in group IV $(\mathrm{p}<0.001)$. The mean event-free survival time decreased as ORS duration and Ts -1 increased (figure 2).

\section{DISCUSSION}

The major finding of this study is that mechanical dyssynchrony has an additional benefit over ORS duration in predicting cardiac events in patients with advanced heart failure. In recently published reports, the use of TDI for the assessment of CRT response has been disappointing. ${ }^{11} 1214$ In the PROSPECT trial, no single echocardiographic measure of dyssynchrony could be recommended to improve patient selection for $\mathrm{CRT}^{12}$ There is a poor agreement for evaluating the magnitude of intraventricular dyssynchrony between TDI and real-time, three-dimensional echocardiography. ${ }^{15}$ Furthermore, mechanical dyssynchrony based on TDI had a limited role in predicting clinical response in patients with narrow ORS width and mechanical dyssynchrony. ${ }^{11}$ In light of these reports, the question remains whether or not TDI is the appropriate diagnostic tool for identifying dyssynchrony in patients with advanced systolic heart failure. Therefore, the ORS duration remains as the dyssynchrony surrogate to identifying patients appropriate for CRT. However, about $10 \%$ of patients with heart failure meet the current criteria for CRT. ${ }^{16}$ Furthermore, most trials have demonstrated that approximately $30-40 \%$ of these 
Table 2 Cox-regression hazards ratio (HR) in univariate and multivariate analysis for predicting cardiac events or death

\begin{tabular}{|c|c|c|c|c|c|c|}
\hline & \multicolumn{3}{|c|}{ Univariate analysis } & \multicolumn{3}{|c|}{ Multivariate analysis } \\
\hline & HR & $95 \% \mathrm{Cl}$ & $\bar{p}$ Value & HR & $95 \% \mathrm{Cl}$ & p Value \\
\hline Age & 1.03 & 1.00 to 1.05 & 0.028 & & & \\
\hline Sex & 1.11 & 0.68 to 1.80 & 0.674 & & & \\
\hline QRS duration ( $\geq 120 \mathrm{~ms}$ ) & 1.70 & 1.05 to 2.76 & 0.032 & 1.88 & 1.07 to 3.29 & 0.028 \\
\hline Ischaemic aetiology & 1.93 & 1.15 to 3.25 & 0.013 & & & \\
\hline Diabetes mellitus & 1.43 & 0.90 to 2.29 & 0.134 & & & \\
\hline Serum creatinine & 1.14 & 1.03 to 1.26 & 0.013 & 1.24 & 1.09 to 1.43 & 0.002 \\
\hline Use of $\beta$ blocker & 0.53 & 0.31 to 0.89 & 0.017 & 0.40 & 0.22 to 0.74 & 0.003 \\
\hline End-systole volume & 1.01 & 1.00 to 1.01 & 0.065 & & & \\
\hline End-diastole volume & 1.00 & 1.00 to 1.01 & 0.062 & & & \\
\hline LV ejection fraction & 0.98 & 0.95 to 1.02 & 0.286 & & & \\
\hline $\mathrm{E} / \mathrm{e}^{\prime}$ & 1.04 & 1.01 to 1.06 & 0.005 & 1.04 & 1.01 to 1.07 & 0.009 \\
\hline MR $\left(E R O \geq 0.2 \mathrm{~cm}^{2}\right)$ & 1.53 & 0.94 to 2.45 & 0.085 & & & \\
\hline Ts -1 (>65 ms) & 2.92 & 1.80 to 4.73 & 0.000 & 2.37 & 1.39 to 4.04 & 0.002 \\
\hline
\end{tabular}

ERO, effective regurgitation orifice area; MR, mitral regurgitation; LV, left ventricular; Ts-I, time difference between the septal and lateral wall.

patients are considered non-responders clinically or based on echocardiographic remodelling. ${ }^{2} 1718$ Therefore, the actual number of patients who benefit from CRT is quite small relative to that of the number of people with heart failure. ${ }^{10}$ Mechanical dyssynchrony itself is associated with a poor prognosis in heart failure $^{19}$ and has a greater sensitivity in achieving both clinical and echocardiographic benefits than electrical dyssynchrony after CRT in many studies. ${ }^{8}{ }^{20-26}$ Not all studies are consistent with our study. However, most studies have shown that mechanical dyssynchrony is associated with higher risk of cardiac events, independent of the ORS duration and LVEF in heart failure, or even patients undergoing coronary bypass surgery. ${ }^{27}$

The most basic question that remains unanswered is how exactly to define the marker of dyssynchrony and who will be most likely to respond to CRT. Therefore, the need for better selection criteria for CRT and better imaging modalities to assess more precisely dyssynchrony has been emphasised. ${ }^{8} 17$ The prognostic value of a prolonged ORS duration has been known for several years. This electrocardiographic evidence of conduction delay has been considered a marker of ventricular dyssynchrony and associated with a higher incidence of cardiac events or mortality. ${ }^{2829}$ It is generally accepted that the ORS duration

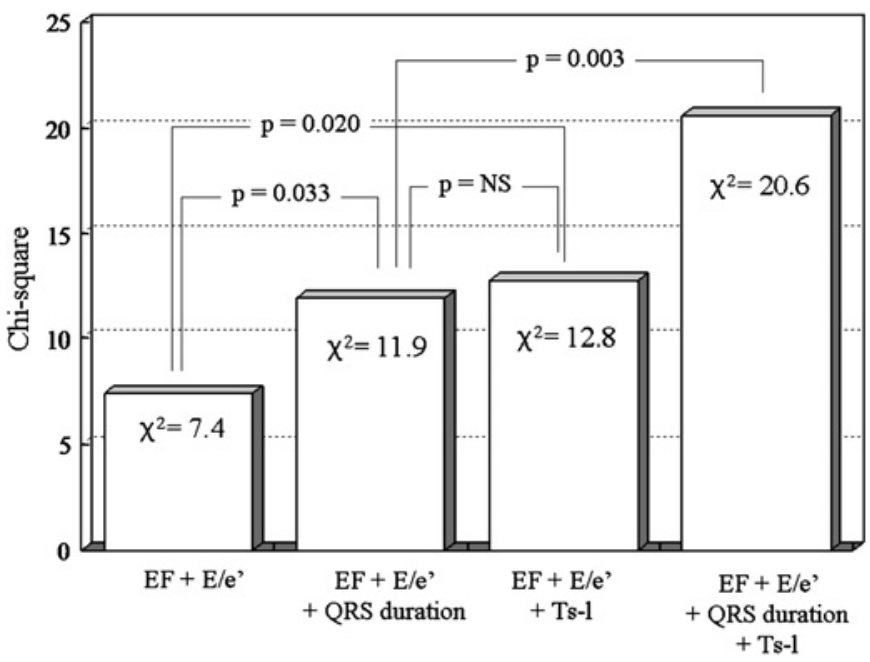

Figure 1 Prognostic power of QRS duration and mechanical dyssynchrony to predict cardiac events. EF, ejection fraction. alone may not effectively characterise mechanical dyssynchrony. ${ }^{1}$ We still have a poor understanding of the interaction between mechanical dyssynchrony and electrical delay. TDI analysis showed that mechanical dyssynchrony was present in nearly half of patients with heart failure with normal ORS duration. $^{30}$ In this study, electrical and mechanical dyssynchrony were shown in $31.1 \%$ and $42.5 \%$. We previously demonstrated that mechanical dyssynchrony predicts cardiac events, even in patients with a normal ORS duration. ${ }^{19}$ Although the prognostic power of mechanical dyssynchrony and the ORS duration assessed by $\chi^{2}$ and AUCs was not different, mechanical dyssynchrony had an additional benefit and was independently associated with cardiac events in patients with systolic heart failure.

The response to CRT is another point of mechanical dyssynchrony because the response is multifactorial, largely dependent on mechanical dyssynchrony and myocardial scar from a prior infarct, inappropriate lead position and suboptimal device programming. Our study was not designed to assess the

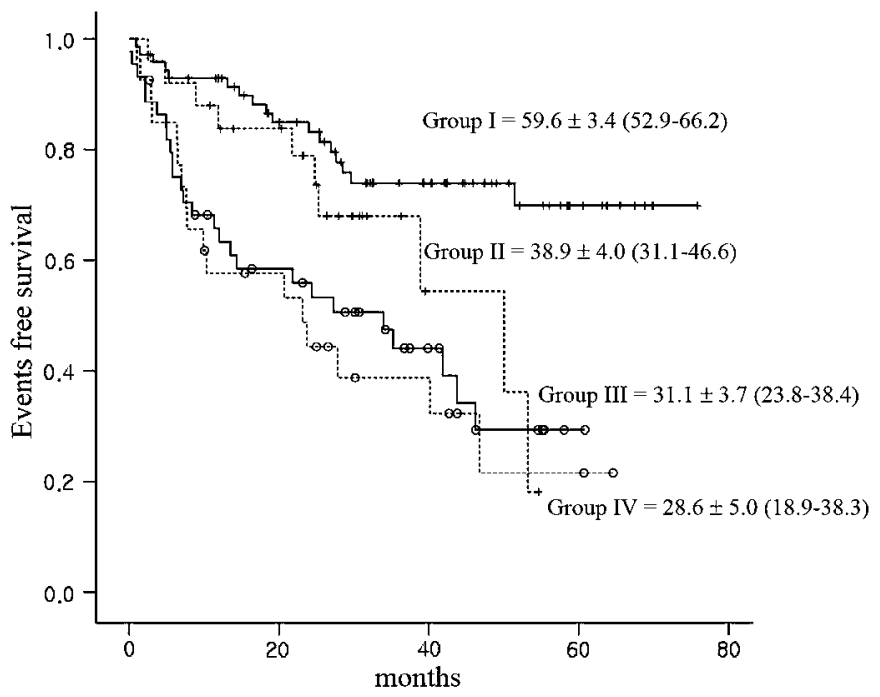

Figure 2 Kaplan-Meier analysis for event free survival. Group I: ORS duration $<120 \mathrm{~ms}$ and Ts $-\mathrm{I}<65 \mathrm{~ms}$, Group II: ORS duration $\geq 120 \mathrm{~ms}$ and Ts $-\mathrm{I}<65 \mathrm{~ms}$, Group III: QRS duration $<120 \mathrm{~ms}$ and Ts $-\mathrm{I} \geq 65 \mathrm{~ms}$, Group IV: QRS duration $\geq 120 \mathrm{~ms}$ and Ts $-\mathrm{I} \geq 65 \mathrm{~ms}$. Data are presented as mean $\pm \mathrm{SE}(\mathrm{CI})$. 
predictive values of dyssynchrony for the response of CRT. Large prospective studies are still needed to determine which parameter of dyssynchrony is best for CRT.

This study has several limitations. The topic is not entirely new. Recent large clinical trials have demonstrated that mechanical dyssynchrony using echocardiography is unsuited for clinical use in CRT selection. In this study, however, echocardiographic techniques in evaluating dyssynchrony are still valuable in predicting adverse events in patients with advanced heart failure. This study is a single-centre, observational study. Furthermore, the number in the study population and the number of patients with hard events was relatively low for identifying various predictors of heart failure.

Competing interests None

Ethics approval This study was conducted with the approval of the institutional review board of Hallym University.

Provenance and peer review Not commissioned; externally peer reviewed.

\section{REFERENCES}

1. Kashani A, Barold SS. Significance of QRS complex duration in patients with heart failure. J Am Coll Cardiol 2005:46:2183-92.

2. Bax JJ, Bleeker GB, Marwick TH, et al. Left ventricular dyssynchrony predicts response and prognosis after cardiac resynchronization therapy. J Am Coll Cardiol 2004:44:1834-40.

3. Molhoek SG, Van EL, Bootsma M, et al. QRS duration and shortening to predict clinical response to cardiac resynchronization therapy in patients with end-stage heart failure. Pacing Clin Electrophysiol 2004;27:308-13.

4. Achilli A, Sassara M, Ficili S, et al. Long-term effectiveness of cardiac resynchronization therapy in patients with refractory heart failure and "narrow". QRS J Am Coll Cardiol 2003;42:2117-24.

5. Yu CM, Fung JW, Zhang 0 , et al. Tissue Doppler imaging is superior to strain rate imaging and postsystolic shortening on the prediction of reverse remodeling in both ischemic and nonischemic heart failure after cardiac resynchronization therapy. Circulation 2004;110:66-73.

6. Yu CM, Chau E, Sanderson JE, et al. Tissue Doppler echocardiographic evidence of reverse remodeling and improved synchronicity by simultaneously delaying regional contraction after biventricular pacing therapy in heart failure. Circulation 2002:105:438-45

7. Sogaard P, Egeblad H, Kim WY, et al. Tissue Doppler imaging predicts improved systolic performance and reversed left ventricular remodeling during long-term cardiac resynchronization therapy. J Am Coll Cardiol 2002;40:723-30.

8. Bax JJ, Molhoek SG, van EL, et al. Usefulness of myocardial tissue Doppler echocardiography to evaluate left ventricular dyssynchrony before and after biventricular pacing in patients with idiopathic dilated cardiomyopathy. Am J Cardiol 2003;91:94-7.

9. Breithardt $\mathbf{O A}$, Breithardt G. Quest for the best candidate: how much imaging do we need before prescribing cardiac resynchronization therapy? Circulation 2006;113:926-8.

10. Beshai JF, Khunnawat C, Lin AC. Mechanical dyssynchrony from the perspective of a cardiac electrophysiologist. Curr Opin Cardiol 2008;23:447-51.
11. Beshai JF, Grimm RA, Nagueh SF, et al. Cardiac-resynchronization therapy in heart failure with narrow QRS complexes. N Engl J Med 2007;357:2461-71.

12. Chung ES, Leon AR, Tavazzi $L$, et al. Results of the Predictors of Response to CRT (PROSPECT) trial. Circulation 2008:117:2608-16.

13. Bleeker GB, Mollema SA, Holman ER, et al. Left ventricular resynchronization is mandatory for response to cardiac resynchronization therapy: analysis in patients with echocardiographic evidence of left ventricular dyssynchrony at baseline. Circulation 2007;116:1440-8.

14. Marwick TH. Hype and hope in the use of echocardiography for selection for cardiac resynchronization therapy: the Tower of Babel revisited. Circulation 2008;117:2573-6.

15. Burgess MI, Jenkins C, Chan J, et al. Measurement of left ventricular dyssynchrony in patients with ischaemic cardiomyopathy: a comparison of real-time threedimensional and tissue Doppler echocardiography. Heart 2007;93:1191-6.

16. Willerson JT, Kereiakes DJ. Cardiac resynchronization therapy: helpful now in selected patients with CHF. Circulation 2004;109:308-9.

17. Abraham WT, Fisher WG, Smith AL, et al. Cardiac resynchronization in chronic heart failure. N Engl J Med 2002;346:1845-53.

18. Cleland JG, Daubert JC, Erdmann E, et al. The effect of cardiac resynchronization on morbidity and mortality in heart failure. N Engl J Med 2005;352:1539-49.

19. Cho GY, Song JK, Park WJ, et al. Mechanical dyssynchrony assessed by tissue Doppler imaging is a powerful predictor of mortality in congestive heart failure with normal QRS duration. J Am Coll Cardiol 2005:46:2237-43.

20. Bax JJ, Abraham T, Barold SS, et al. Cardiac resynchronization therapy: Part 2-issues during and after device implantation and unresolved questions. J Am Coll Cardiol 2005:46:2168-82

21. Bax JJ, Abraham T, Barold SS, et al. Cardiac resynchronization therapy: Part 1-issues before device implantation. J Am Coll Cardiol 2005:46:2153-67.

22. Suffoletto MS, Dohi K, Cannesson M, et al. Novel speckle-tracking radial strain from routine black-and-white echocardiographic images to quantify dyssynchrony and predict response to cardiac resynchronization therapy. Circulation 2006;113:960-8

23. Notabartolo D, Merlino JD, Smith AL, et al. Usefulness of the peak velocity difference by tissue Doppler imaging technique as an effective predictor of response to cardiac resynchronization therapy. Am J Cardiol 2004;94:817-20.

24. Sogaard P, Egeblad H, Pedersen AK, et al. Sequential versus simultaneous biventricular resynchronization for severe heart failure: evaluation by tissue Doppler imaging. Circulation 2002;106:2078-84.

25. Turner MS, Bleasdale RA, Vinereanu D et al. Electrical and mechanical components of dyssynchrony in heart failure patients with normal QRS duration and left bundle-branch block: impact of left and biventricular pacing. Circulation 2004;109:2544-9.

26. Van de Veire NR, Bleeker GB, De SJ, et al. Tissue synchronisation imaging accurately measures left ventricular dyssynchrony and predicts response to cardiac resynchronisation therapy. Heart 2007:93:1034-9.

27. Penicka $\mathbf{M}$, Bartunek J, Lang 0 , et al. Severe left ventricular dyssynchrony is associated with poor prognosis in patients with moderate systolic heart failure undergoing coronary artery bypass grafting. J Am Coll Cardiol 2007:50:1315-23.

28. Grines CL, Bashore TM, Boudoulas $\mathrm{H}$, et al. Functional abnormalities in isolated left bundle branch block. The effect of interventricular asynchrony. Circulation 1989;79:845-53.

29. Iuliano S, Fisher SG, Karasik PE, et al. QRS duration and mortality in patients with congestive heart failure. Am Heart J 2002;143:1085-91.

30. Yu CM, Lin $\mathrm{H}$, Zhang $\mathrm{O}$, et al. High prevalence of left ventricular systolic and diastolic asynchrony in patients with congestive heart failure and normal QRS duration. Heart 2003;89:54-60. 